\title{
Energy Metabolism Disturbances in Cell Models of PARK2 CNV Carriers with ADHD
}

\author{
Viola Stella Palladino ${ }^{1}$, Andreas G. Chiocchetti ${ }^{2}$, Lukas Frank ${ }^{1}$, Denise Haslinger ${ }^{2}$, \\ Rhiannon McNeill ${ }^{3}$, Franziska Radtke ${ }^{4}$, Andreas Till ${ }^{5}{ }^{-}$, Simone Haupt ${ }^{6,7}$, Oliver Brüstle ${ }^{5}$, \\ Katharina Günther ${ }^{8,9}{ }^{\circledR}$, Frank Edenhofer ${ }^{8}$, Per Hoffmann ${ }^{10}$, Andreas Reif ${ }^{1,+}$ and \\ Sarah Kittel-Schneider $1,3, *,+\mathbb{C}$
}

1 Department of Psychiatry, Psychotherapy and Psychosomatic Medicine, University Hospital, Goethe University, D-60528 Frankfurt, Germany; viola.stella.palladino@outlook.it (V.S.P.); lukas.ms.frank@googlemail.com (L.F.); andreas.reif@kgu.de (A.R.)

2 Department of Child and Adolescent Psychiatry and Psychotherapy, University Hospital Frankfurt, D-60528 Frankfurt, Germany; Andreas.Geburtig-Chiocchetti@kgu.de (A.G.C.);

Denise.haslinger@kgu.de (D.H.)

3 Department of Psychiatry, Psychotherapy and Psychosomatic Medicine, University Hospital, University of Würzburg, D-97080 Würzburg, Germany; McNeill_R@ukw.de

4 Department of Child and Adolescent Psychiatry, Psychotherapy and Psychosomatic Medicine, University Hospital, University of Würzburg, D-97080 Würzburg, Germany; Radtke_F@ukw.de

5 Institute of Reconstructive Neurobiology, LIFE \& BRAIN Center, University of Bonn Medical Faculty \& University Hospital Bonn, D-53127 Bonn, Germany; andy.till.0042@gmail.com (A.T.); oliver.bruestle@uni-bonn.de (O.B.)

6 LIFE \& BRAIN GmbH, Cellomics Unit, D-53127 Bonn, Germany; shaupt@lifeandbrain.com

7 Department 75, Transfer, University of Cologne, 50923 Cologne, Germany

8 Institute of Molecular Biology \& CMBI, University of Innsbruck, AT-6020 Innsbruck, Austria; Katharina.Guenther@uibk.ac.at (K.G.); frank.edenhofer@uibk.ac.at (F.E.)

9 Spinal Cord Injury and Tissue Regeneration Center Salzburg (SCI-TReCS), Paracelsus Medical University Salzburg, AT-5020 Salzburg, Austria

10 Institute of Humane Genetics, LIFE \& BRAIN Center, University of Bonn Medical Faculty \& University Hospital Bonn, D-53127 Bonn, Germany; p.hoffmann@uni-bonn.de

* Correspondence: kittel_s@ukw.de; Tel.: +49-931-201-77100

$\dagger$ These authors contributed equally.

Received: 21 October 2020; Accepted: 13 December 2020; Published: 18 December 2020

Abstract: The main goal of the present study was the identification of cellular phenotypes in attention-deficit-/hyperactivity disorder (ADHD) patient-derived cellular models from carriers of rare copy number variants (CNVs) in the PARK2 locus that have been previously associated with ADHD. Human-derived fibroblasts (HDF) were cultured and human-induced pluripotent stem cells (hiPSC) were reprogrammed and differentiated into dopaminergic neuronal cells (mDANs). A series of assays in baseline condition and in different stress paradigms (nutrient deprivation, carbonyl cyanide $\mathrm{m}$-chlorophenyl hydrazine (CCCP)) focusing on mitochondrial function and energy metabolism (ATP production, basal oxygen consumption rates, reactive oxygen species (ROS) abundance) were performed and changes in mitochondrial network morphology evaluated. We found changes in PARK2 CNV deletion and duplication carriers with ADHD in PARK2 gene and protein expression, ATP production and basal oxygen consumption rates compared to healthy and ADHD wildtype control cell lines, partly differing between HDF and mDANs and to some extent enhanced in stress paradigms. The generation of ROS was not influenced by the genotype. Our preliminary work suggests an energy impairment in HDF and mDAN cells of PARK2 CNV deletion and duplication carriers with ADHD. The energy impairment could be associated with the role of PARK2 dysregulation in mitochondrial dynamics. 
Keywords: ADHD; hiPSC; PARK2; mitochondria; disease modelling

\section{Introduction}

Attention-deficit/hyperactivity disorder (ADHD) is a very heterogeneous disorder, with a broad spectrum of type and severity of symptoms that interfere with personal functioning and negatively impacts social and occupational activities [1,2]. The general population prevalence of ADHD has been described to be between $4-7 \%$ in childhood, and $2-4 \%$ in the adult population worldwide [3]. The genetic contribution to ADHD has been estimated between 70-80\% [4,5], whereas environment is suggested to explain about $22 \%$ of ADHD variance [6-9]. In the past decades, the development of whole-genome scanning methods allowed to clarify the major contribution of copy number variants (CNVs) to genetic variance. CNVs are large, genomic structural variations that comprise deletions, duplications, triplications, and translocations in comparison to a reference genome [10]. Several rare CNV have been associated with ADHD [11-13]. There is evidence that the risk for ADHD fits a polygenic liability threshold model. This means that individuals carrying rare large CNVs could develop ADHD by only carrying a lower number of multiple common genetic risk variants [14]. A genome-wide analysis of rare CNVs conducted by Jarick and colleagues specifically detected CNVs in the PARK2 $(=P R K N)$ genetic locus, implicating this target as a candidate gene for ADHD [15]. PARK2 (also known as Parkin/PRKN, a protein with E3 Ubiquitin ligase function), in concert with the Ser/Thr protein kinase PINK1, plays a pivotal role in the regulation of the mitochondria quality control (MQC) system directing processes such as mitophagy (i.e., selective autophagy-mediated degradation of mitochondria), fusion and fission, biogenesis and mitochondrial transport, and is thus involved in the cellular energy balance and oxidative stress response [16]. Different stimuli, both physiological and pathological, can lead to PINK1 accumulation on the mitochondrial outer membrane where it can recruit and activate cytosolic PARK2 by phosphorylation. PARK2 is then able to flag several proteins expressed on the mitochondrial surface and in the cytoplasm with ubiquitin tags, thus marking them for degradation by the ubiquitine-proteasome system or by autophagy [17]. Acting together, PARK2 and PINK1 represent an internal sensor system for disparate cellular homeostasis perturbations [18]. In addition to mitochondria's well-known role in energy production, they play a pivotal role in general cellular metabolism, intracellular calcium signaling, generation of ROS and stress responses [19]. In the last years, studies have implicated mitochondrial (mt) dysfunction in ADHD in that increased oxidative markers [20], reduced oxygen consumption, and ATP production as well as increased levels of superoxide radicals have been reported [21].

The role of PARK2 mutations in Parkinson's patients are relatively well studied and recent studies also utilize human-induced pluripotent stem cell (hiPSC)-derived neuronal cells [22,23] in this context, but no data are available yet regarding the functional consequences of PARK2 CNVs associated with ADHD in human-derived cellular models.

In this study, we investigated two different cell models; human fibroblast cell lines (HDF) and as well preliminary data from hiPSC-derived midbrain-derived dopaminergic neurons (mDANs). Cells were derived from adult ADHD patients carrying PARK2 CNVs (deletion and duplication) in comparison to healthy and ADHD wildtype (WT) controls. We conducted experiments focusing on mitochondrial function and energy metabolism. The MQC system is responsible for basal and normal cellular functions but also plays an important role after cellular homeostasis disturbances [18]. Thus, we chose to additionally investigate if baseline genotype differences were more pronounced after a nutrient deprivation paradigm ("starvation"). This displays a form of metabolic cellular stress that has been shown to induce increased PARK2 expression [24]. We also applied pharmacological treatment using carbonyl cyanide m-chlorophenyl hydrazine (CCCP), an ionophore that depolarizes the mitochondrial membrane thus triggering PINK1 accumulation and subsequent mitochondrial degradation [25]. Finally, we performed a series of assays focusing on mitochondrial function 
and energy metabolism (ATP production, basal oxygen consumption rates, reactive oxygen species (ROS) abundance).

\section{Methods}

\subsection{Neuropsychiatric Assessment and Genotyping}

Patients were recruited in 2013 at the Department of Psychiatry, Psychosomatic Medicine and Psychotherapy, University Hospital of Würzburg, Germany within a previously published sample [15] (see Table S1 and Supplementary Material 1). The healthy controls were recruited among hospital staff and did not report a history of mental disorders, acute or chronic infections, or severe somatic diseases. Only study participants who gave written informed consent were enrolled in the study, which complied with the latest Declaration of Helsinki and was approved by the Ethics Committee of the University of Würzburg (votum no 96/10). Participants were also examined for early signs of Parkinson's disease by Unified Parkinson's Disease Rating Scale (UPDRS), Non-Motor Symptom assessment scale for Parkinson's disease (PD NMS), and the sniffing test to assess olfactory function. Additionally, Substantia nigra volume was assessed by ultrasound and IQ was measured using the MWT-B (Multiple-Choice Vocabulary Intelligence Test, Mehrfachwahl-wortschatz-test, verbal intelligence) [26]. We recruited four ADHD patients and two healthy controls for skin punch biopsies. From three ADHD patients that were known as $\mathrm{CNV}$ carriers from the previously published sample [15] and one healthy wildtype control, we further reprogrammed HDF into hiPSC and differentiated them into mDANs (one adult ADHD PARK2 CNV duplication risk-carrier = PARK2CNV_DUP/ADHD, one adult ADHD PARK2 CNV deletion risk-carrier $=$ PARK2CNV_DEL_A/ADHD and one healthy CNV non-risk carrier = WT_A/HEALTHY, see Table S1). From the three additional participants fibroblasts and hiPSC were generated (one adult ADHD PARK2 deletion carrier = PARK2_DEL_B/ADHD, one adult ADHD patient with the wildtype variation $=$ WT/ADHD and another healthy wildtype control $=$ WT_B/HEALTHY) (see Table S1). For our experiments, we used (i) HDF from all six participants to have a larger number and the generated (ii) mDANs from the described three participants. We obtained venous blood samples from the participants and DNA was isolated from EDTA-monovettes (Sarstedt, Nümbrecht, Germany) by a de-salting method [27]. DNA concentration and quality were assessed by spectrophotometric measurement (Infinite 200 PRO-Tecan, Männedorf, Switzerland). The presence of the PARK2 risk-CNV was confirmed by Illumina Infinium Omni2.5-8 bead array analysis.

\subsection{Skin Biopsies and Fibroblast Primary Cultures}

Skin biopsies were taken by medically trained personnel (SKS) under local anesthesia (Scandicain, AstraZeneca, Wedel, Germany) using a skin puncher $\left(3 \mathrm{~mm}^{2}\right)$ and fibroblast cultures were generated after standard procedure (see Supplementary Material 1).

\section{3. hiPSCs Generation and Pluripotency Assays}

CytoTune-IPs 2.0 Sendai Reprogramming Kit (Invitrogen, Carlsbad, CA, USA) was used to reprogram the fibroblasts into hiPSCs following the manufacturer's protocol (see Supplementary Material). Embryoid body (EB) assay was performed as well as immunostaining and PCR for pluripotency markers in hiPSC and EBs, see Supplementary Material 1 and Figure S1 and Tables S2 and S3).

\subsection{Differentiation of hiPSCs into Neurons with a Midbrain Dopamine Like Phenotype}

Dopaminergic differentiation was chosen because PARK2 genetic mutation are commonly causal for familial Parkinson's disease and here neurodegeneration in dopaminergic cells plays an important role [28]. On the other hand, the cells in our study were generated from ADHD patients and in ADHD as well dopaminergic dysfunction is suspected [29]. In addition, methylphenidate as the most commonly used ADHD drug is a norepinephrine- and dopamine reuptake inhibitor [30]. The neural differentiation was performed employing a protocol adapted from Kriks et al. [31] as described 
elsewhere [32]. For basal characterization of dopaminergic markers and dopamine production see Supplementary Material 1 and Figure S2A-C.

\subsection{Immunofluorescence and Mitostaining}

For immunofluorescence assays cells were grown on Matrigel-coated coverslips. For the analysis of mitochondrial network morphology, fibroblast cells were grown on coverslips and mitochondria were stained using $400 \mathrm{nM}$ MitoTracker ${ }^{\circledR}$ Red CMXRos (ThermoFisher Scientific, Waltham, MA, USA) and imaged with Zeiss Axio Observer.Z1 microscope with ApoTome function (Zeiss, Oberkochen, Germany). Images were analyzed by a semi-automated analysis with Fiji ImageJ software as described elsewhere [33]. Shape descriptors considered were aspect ratio (AR): major_axis/minor_axis and Form factor $(\mathrm{FF})$ : (perimeter2/( $4 \pi \times$ area)) (details see Supplementary Material 1$)$.

\subsection{Molecular Karyotyping}

Genomic DNA from cell lines was extracted with the DNeasy kit (Quiagen, Hilden, Germany) and analyzed on an Illumina Infinium Omni2.5-8 bead array at the Institute of Human Genetics, LIFE\&BRAIN, University of Bonn (for further details see Supplementary Material 1).

\subsection{RNA Extraction, Two-Steps Reverse Transcription PCR (RT-PCR), and Quantitative RT-PCR (RT-qPCR)}

RNA was isolated using RNeasy-Plus Mini Kit (Qiagen, Hilden, Germany) according to manufacturer's instructions. RNA quality and absence of gDNA contamination was measured using the Standard Sensitivity RNA Analysis Kit with Fragment Analyzer (Advanced Analytical, Agilent, Santa Clara, CA, USA). Because of the low gene expression of PARK2 in fibroblasts, samples were pre-amplified before PARK2 gene expression analysis. Pre-amplification of the target genes was performed with TaqMan PreAmp Master Mix Kit (ThermoFisher Scientific, Waltham, MA, USA) following the manufacturer's protocol (for predesigned primer and probe sets see Table S2). Quantitative RT-PCR (RT-qPCR) primers were designed to be intron spanning with NCBI Primer-Blast with the size of the respective intron being greater than $1 \mathrm{kbp}$ (sequence on Table S1 and Supplementary Material 1).

\subsection{Protein Concentration}

Protein concentration from whole cell lysate was determined with ADV02 assay (Cytoskeletron Inc, Denver, CO, USA) and absorbance was measured at $600 \mathrm{~nm}$ wavelength by the Infinite M200 PRO microplate reader (Tecan, Männedorf, Switzerland). PARK2 protein levels were measured with Human Parkin SimpleStep (Enzyme-linked Immunosorbent Assay) ELISA Kit (Abcam, Cambridge, UK) following the manufacturer's instructions (for further details see Supplementary Material 1).

\subsection{Stressor Paradigms}

For the subsequent experimental procedures, the cell lines were divided into three groups. The "baseline group" was cultured with standard maintenance media; DMEM with 10\%FBS for fibroblasts, and complete neurobasal medium for mDANs. The "starvation group" was subjected to a 24-h serum nutrient deprivation paradigm; DMEM-only for fibroblasts, and neurobasal medium without B27 supplementation for mDANs. The "CCCP group" was cultured with standard culturing media supplemented with $10 \mu \mathrm{M}$ carbonyl cyanide 3-chlorophenylhydrazone (Sigma-Aldrich, Taufkirchen, Germany) for $24 \mathrm{~h}$. For experimental procedures cultured cells from the same line were randomly assigned to one of the treatment groups. Cells were used in similar passage numbers (for further details see Supplementary Material 1). 


\subsection{ATP Production, Oxygen Consumption, and ROS Production}

All assays were conducted after standard procedure (see Supplementary Material 1) using the Plate reader Infinite 200 PRO (Tecan, Männedorf, Switzerland). ATP production was analyzed using the ATPlite Luminescence Assay System (PerkinElmer, Walluf, Germany) according to manufacturer's instructions. ROS production was measured using the DCFDA/H2DCFDA-Cellular Reactive Oxygen Species Detection Assay Kit (Abcam, Cambridge, GB, USA), according to a protocol suggested by the manufacturer for $24 \mathrm{~h}$ of treatment.

\subsection{Data Analysis}

For each experiment we report the single values of each individual replicate that were also used for statistical analysis. Data were analyzed using SPSS (V22, IBM, Armonk, NY, USA). Data were tested for normal distribution by Kolmogorov-Smirnov and Shapiro-Wilk test and parametric or non-parametric tests were applied appropriately. Exploratory one-way ANOVA or two-way ANOVA as well as repeated measures ANOVA (univariate analysis of variance) were used and Kruskal-Wallis test or Mann-Whitney test were performed. If statistical analysis showed significant difference, post-hoc testing was performed (Tukey HD test). Additionally, effect size was calculated (Cohen's d or Partial eta squares). Correlation between values obtained from HDF and values obtained from mDANs was calculated by bivariate correlation and Pearson's $r$ reported. The level of significance was set at $p=0.05$ because of the exploratory approach and we did therefore not correct for multiple testing. For the mDANs, as only $n=1$ per group was available, only exploratory and descriptive results from the repeated experiments and means of technical replicates are reported. Graphs were plotted using GraphPad Prism 5.01 (GraphPad Software, San Diego, CA, USA). Standard curves were rendered by using CurveExpert 1.4 (https://curveexpert.software.informer.com/1.4/).

\section{Results}

\subsection{Confirmation of PARK2 CNVs}

PARK2 CNV status was confirmed by an Illumina Infinium Omni2.5-8 bead array that showed PARK2 CNVs, spanning exon 2 of transcript NM_004562 (Figures S3A and S5) (PARK2 CNV hg19 position: PARK2 CNV DUPLICATION/ADHD chr6:162737426-162882874; PARK2 CNV DELETION/ADHD chr6:162719417-162914986).

\subsection{Neurological and Psychiatric Assessment}

None of the clinical tests regarding early signs of Parkinson's disease was above the clinical threshold in the part III (motor examination) to allow a diagnosis of PD. There were no significant differences between the PARK2 CNV carriers and the controls (see Table S1).

\subsection{Evaluation of Mitochondrial Network Morphology}

Given the known physiological role of PARK2, we assessed whether the presence of a PARK2 CNV might exert downstream effects on biological processes regulated by the PARK2-PINK1 interaction [34]. Two main parameters were considered in our study using the HDF. First, the aspect ratio (AR), which mainly is used for a description of the shape of the mitochondria (a value of 1 translates to a circle, numbers $>1$ mean a more elongated shape). Second, the form factor (FF), which describes the mitochondrial network branching (high values imply a tubular network and lower values stand for a more fragmented network) $[28,35]$.

Using the mean AR and FF values of the mitochondria present in each HDF (15 cells per cell line/condition) a significant effect of treatment (nutrient deprivation) could be seen for both the indices $\left(\right.$ AR: $\left.F_{(1,84)}=59.715, p \leq 0.0001, \eta_{p}{ }^{2}=0.416 ; F F: F_{(1,84)}=16.147, p \leq 0.000, \eta_{p}{ }^{2}=0.246\right)$ and the genotype $\left(A R: F_{(2,84)}=40.067, p \leq 0.0001, \eta_{\mathrm{p}}{ }^{2}=0.488\right.$; FF: $\left.F_{(2,84)}=13.713, p \leq 0.000, \eta_{\mathrm{p}}{ }^{2}=0.246\right)$, 
and for the interaction between the two variables (AR: $\mathrm{F}_{(2,84)}=35.441, p \leq 0.0001, \eta_{\mathrm{p}}{ }^{2}=0.458$; FF: $\left.\mathrm{F}_{(2,84)}=22.492, p \leq 0.0001, \eta_{\mathrm{p}}^{2}=0.349\right)$. For both $\mathrm{AR}$ and $\mathrm{FF}$, the main difference was between PARK2 CNV duplication/ADHD versus WT/healthy and WT/ADHD control (Tukey HSD $p \leq 0.0001$ ) and PARK2 CNV deletion-carrier/ADHD (Tukey HSD $p \leq 0.0001$ ). Our findings suggest that the PARK2 CNV duplication carrier/ADHD cells with and without nutrient deprivation might exhibit a pronounced elongated mitochondrial shape and tubular branching compared to PARK2 CNV deletion carrier/ADHD and WTs with and without ADHD (see Figure 1A-C).
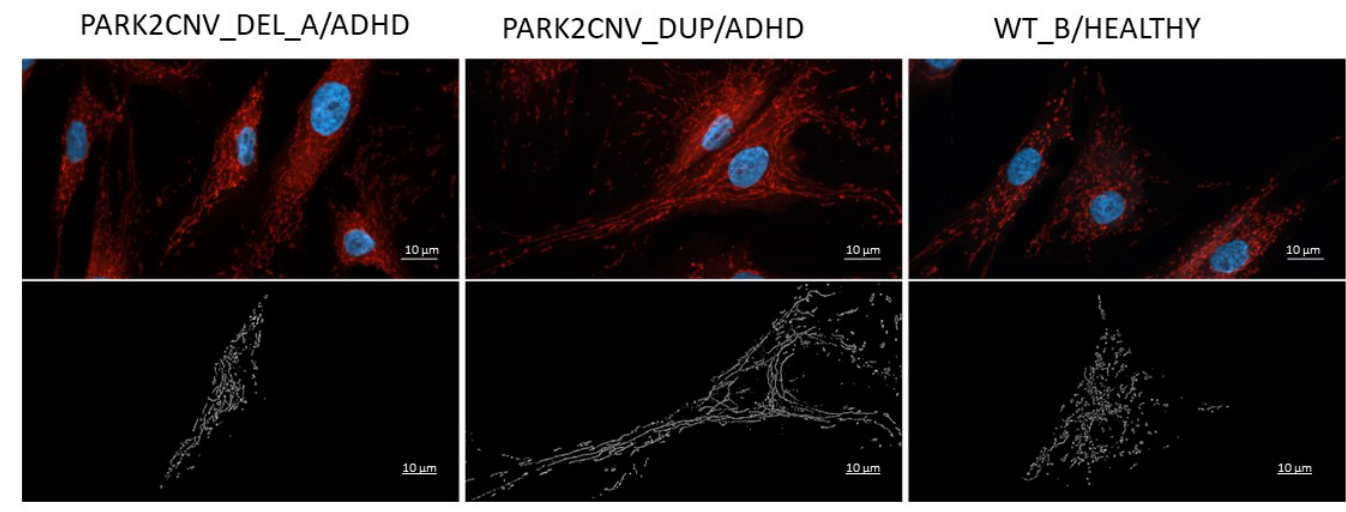

(A)

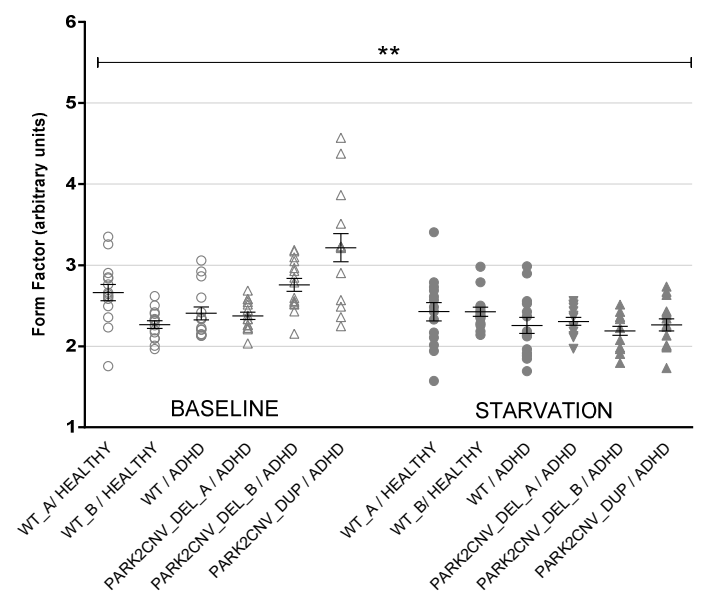

(B)

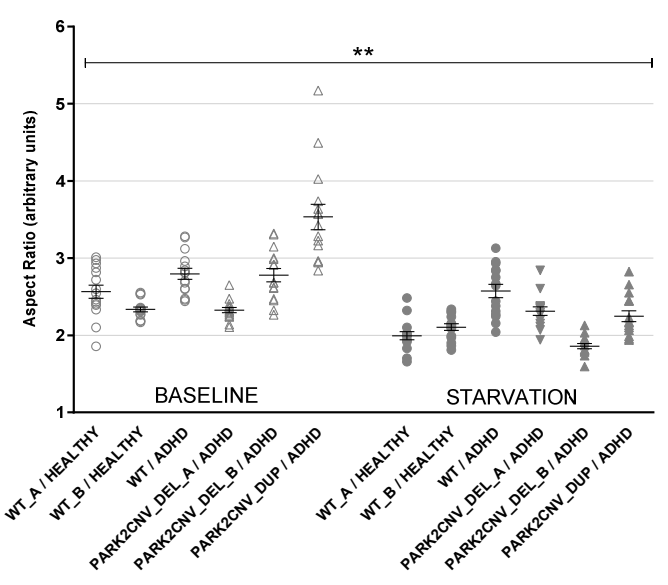

(C)

Figure 1. (A-C): Mitochondrial network analysis. (A) Mitochondrial staining (MitoTracker Red CMXRos) in fibroblast cell lines under baseline conditions, and mask of the semi-automated digital image analysis (Fij//ImageJ) showing different types of network morphology. These images demonstrate a tubular elongated mitochondrial shape (PARK2CNV_DUP/ADHD) and a fragmented round-shaped mitochondrial network (WT_B/HEALTHY and PARK2CNV_DEL_A). Scale bars $10 \mu \mathrm{m}$. Graphical representation of the form factor (FF) (1B) and Aspect ratio (AR) (1C). The values were obtained by analyzing 15 cells per line/condition from two independent experiments. The dots represent the mean AR and FF values of all the mitochondria present in each fibroblast cell. In both cases there seem to be a more elongated mitochondrial shape and tubular branching in the PARK duplication carrier (PARK2CNV_DUP/ADHD) compared to the other cells (PARK2CNV_DEL_A/ADHD, PARK2CNV_DEL_B/ADHD, WT_A.WT_B/HEALTHY as well as WT/ADHD). ANOVA was calculated, level of significance was set at $p=0.05 .{ }^{* *} p \leq 0.01$. Data are shown as mean $\pm \mathrm{SEM}$. 


\subsection{PARK2 Gene and Protein Expression}

We investigated if there were differences in the PARK2 gene expression in fibroblasts due to their genotype and/or after nutrient deprivation (starvation) (Table S4). PARK2 gene expression was generally low in our HDF cultures which was a finding that differed from previous studies [24] and a pre-amplification step was necessary. PARK2 gene expression was nominally lower in HDF cells of the PARK2 deletion carriers in comparison to healthy control and duplication carriers. However, the PARK2 duplication carriers showed a PARK2 gene expression which also was slightly nominally lower in comparison to wildtype healthy and ADHD controls. Statistical analysis using exploratory ANOVA did not show significant differences in PARK2 gene expression between PARK2 deletion and duplication carriers and wildtype controls (with/without ADHD) (Table S4). Nutrient deprivation (=starvation) led to an increase in PARK2 gene expression in PARK2 deletion and duplication carriers, in healthy controls the PARK2 expression remained on the same level after the stress paradigm, however, no significant statistical effect was detected in an exploratory ANOVA analysis (Table S4).

We further assessed Parkin protein levels both in fibroblasts and dopaminergic neuron lines in baseline conditions, after $24 \mathrm{~h}$ of nutrient deprivation and after $24 \mathrm{~h}$ treatment with $10 \mu \mathrm{M}$ carbonyl cyanide 3-chlorophenylhydrazone (CCCP).

Fibroblast cell lines were first explored separately (regarding duplication vs. deletion vs. wildtype) but similar to the PARK2 gene expression levels, as well the PARK2 duplication as the PARK2 deletion carriers showed reduced PARK2 protein levels compared to the wildtype (Figure 2A). An exploratory ANOVA was calculated to assess genotype and treatment effects on PARK2 protein concentration in HDF lines taking the duplication and deletion carriers as one group vs. the wildtype ADHD and healthy control as one group to increase statistical power (Figure 2B). The analysis showed a significant effect of the genotype $\left(\mathrm{F}_{(2,27)}=11.082, p \leq 0.0001, \eta_{\mathrm{p}}{ }^{2}=0.451\right)$ and significant effect of treatment $\left(\mathrm{F}_{(2,27)}=13.810, p \leq 0.0001, \eta_{\mathrm{p}}{ }^{2}=0.506\right)$ but not a significant interaction between the two fixed factors. Post-hoc comparisons using the Tukey HSD on the treatment effect indicated that the treatment difference was mainly driven by CCCP treatment vs. baseline and vs. starvation (both Tukey HSD $p \leq 0.0001$ ) that induced a decrease of PARK2 protein levels. In regard of the genotype, not only fibroblasts lines derived from PARK2 deletion carrier but also PARK2 duplication carrier showed lower levels of PARK2 protein when compared to WT (both Tukey HSD $p \leq 0.0001$ ).

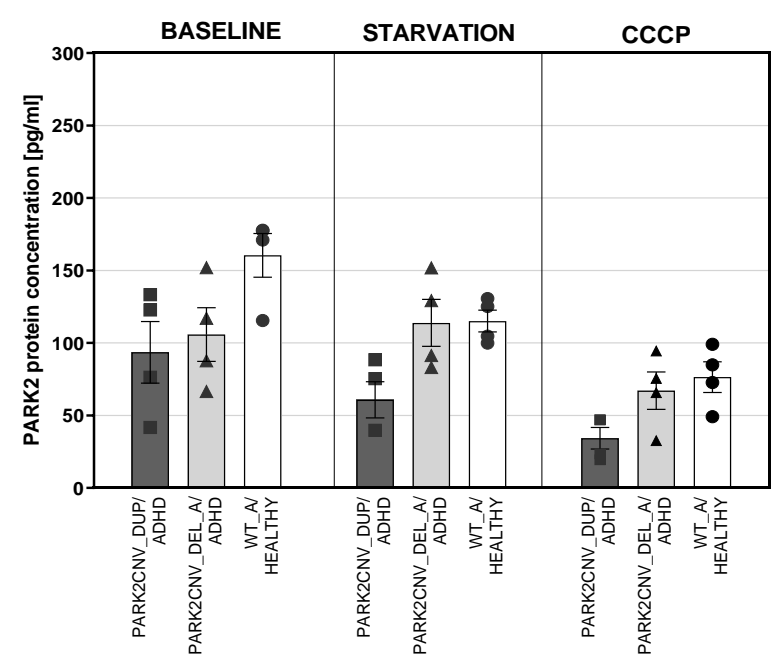

(A)

Figure 2. Cont. 


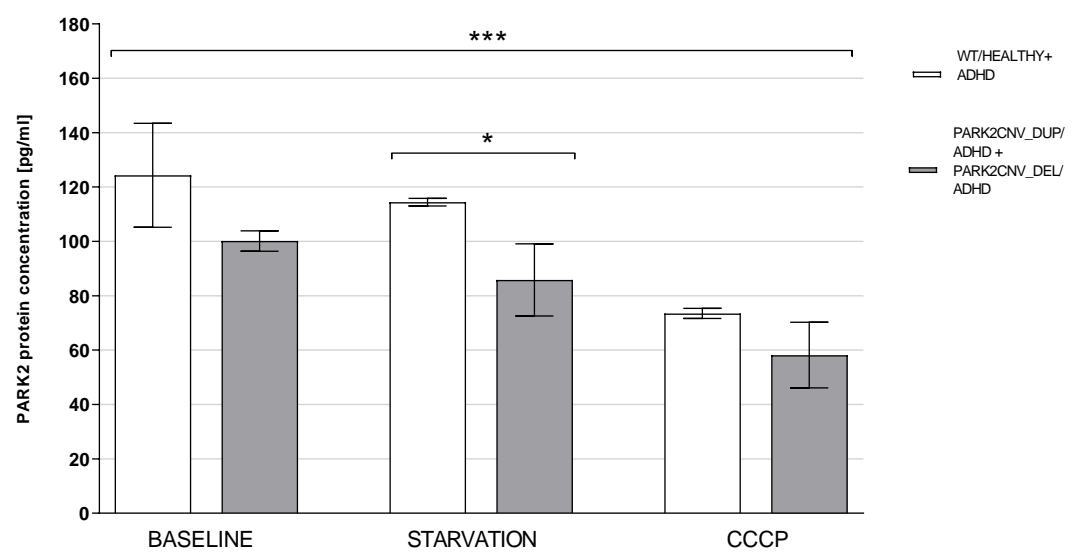

(B)

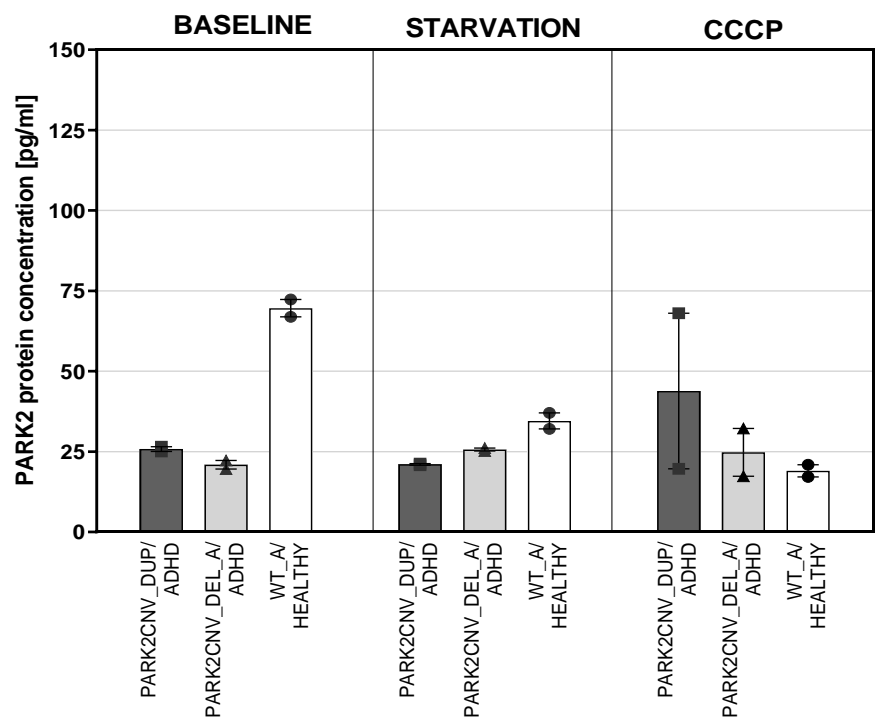

(C)

Figure 2. (A-C): PARK2 protein concentration. PARK2 protein concentration was evaluated in HDF lines $(\mathbf{A}+\mathbf{B})$ and mDANs $(\mathbf{C})$ in baseline conditions and after $24 \mathrm{~h}$ nutrient deprivation (=starvation) stress and after treatment with $10 \mu \mathrm{M}$ CCCP for $24 \mathrm{~h}$. PARK2 protein data were obtained from two independent experiments (HDF, only one experiment in mDAN) with samples measured in duplicates. Data are shown as mean \pm SEM. Exploratory ANOVA was calculated. A show the data from HDF cell lines from PARK2 CNV duplication vs. deletion vs. wildtype healthy control separately, $\mathrm{C}$ shows a combined analysis of HDF data of PARK2 CNV deletion + duplication vs. wildtype ADHD + healthy to increase number of cell lines and statistical power. Level of significance was set at $p=0.05 .{ }^{*} p \leq 0.05$; $* * * \quad p \leq 0.001$.

The measurement of PARK2 protein levels on differentiated dopaminergic neuronal cells (Figure 2C) revealed a significant interaction between genotype and treatment $\left(\mathrm{F}_{(4,9)}=4.819, p=0.024, \eta_{\mathrm{p}}{ }^{2}=0.682\right)$. The wildtype healthy control mDAN line showed increased PARK2 protein levels in comparison to as well PARK2CNV_DUP/ADHD as PARK2CNV_DEL_A/ADHD at baseline (Figure 2B). Also nutrient deprivation as CCCP treatment decreased PARK2 protein concentration in wildtype mDANs. CCCP treatment led to an increase of PARK2 protein levels in PARK2 duplication carrier mDAN (Figure 2C).

\subsection{ATP Levels}

The total cellular ATP concentration was evaluated under baseline conditions, after 24-h starvation stress and after 24-h treatment with $10 \mu \mathrm{M} \mathrm{CCCP}$. The HDF cell lines were first explored separately 
(regarding duplication vs. deletion vs. wildtype) but similar to the other experiments, as well the PARK2 duplication as the PARK2 deletion carriers showed reduced ATP levels/fold changes compared to the wildtype in baseline and after starvation stress. After CCCP treatment the different cell lines showed similar fold changes in comparison to the healthy control at baseline (Figure 3A). In HDF, an exploratory univariate analysis of variance performed on ATP concentration data taking the duplication and deletion carriers as one group vs. the wildtype ADHD and healthy control as one group to increase the statistical power showed a significant difference due to genotype $\left(\mathrm{F}_{(2,44)}=11.462\right.$, $\left.p<0.0001, \eta_{\mathrm{p}}{ }^{2}=0.343\right)$ and treatment $\left(\mathrm{F}_{(2,44)}=43.465, p<0.0001, \eta_{\mathrm{p}}{ }^{2}=0.664\right)$, as well as an interaction effect between the two variables $\left(\mathrm{F}_{(4,44)}=4.666, p=0.003, \eta_{\mathrm{p}}{ }^{2}=0.298\right)$ (Figure 3C). Both stressor treatments appeared to lower the amount of ATP in comparison to the baseline conditions (both Tukey HSD $p<0.0001)$. Genotype differences were mainly observed between the PARK2 CNV deletion carrier/ADHD and WT/Healthy and WT/ADHD (Tukey HSD $p<0.0001$ ), and to a lesser extent between the PARK2 CNV duplication and WT/Healthy and WT/ADHD (Tukey HSD $p=0.064$ ). In both cases it appeared that ADHD/PARK2 CNV carrier cells showed lower levels of ATP compared to WT healthy and ADHD controls, both in basal conditions and after 24-h starvation. However, CCCP treatments appeared to affect all genotypes equally (Figure 3A,B).

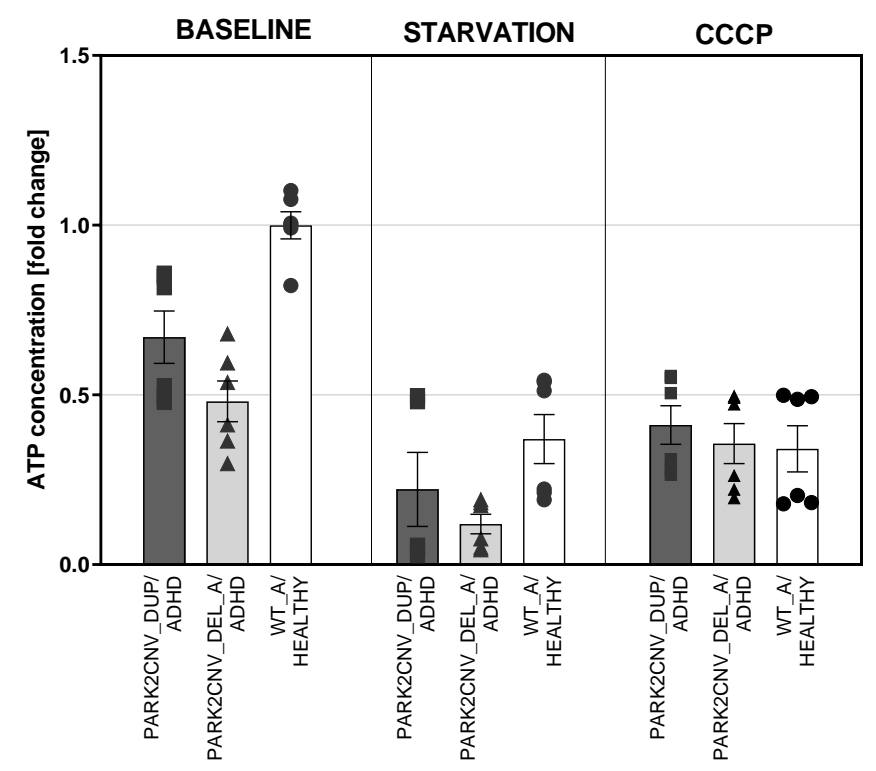

(A)

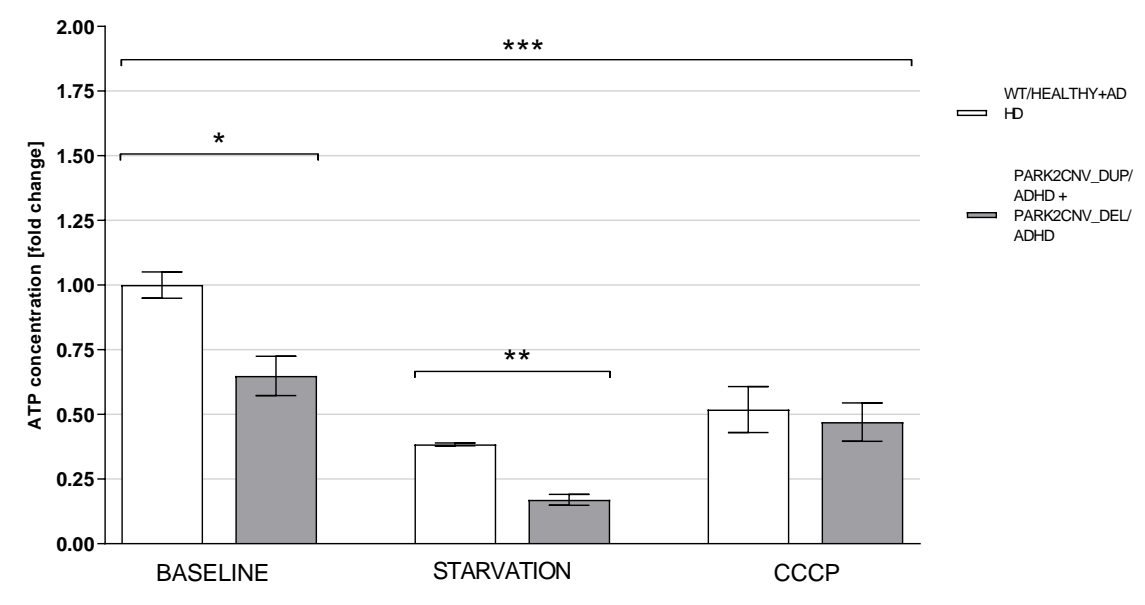

(B)

Figure 3. Cont. 


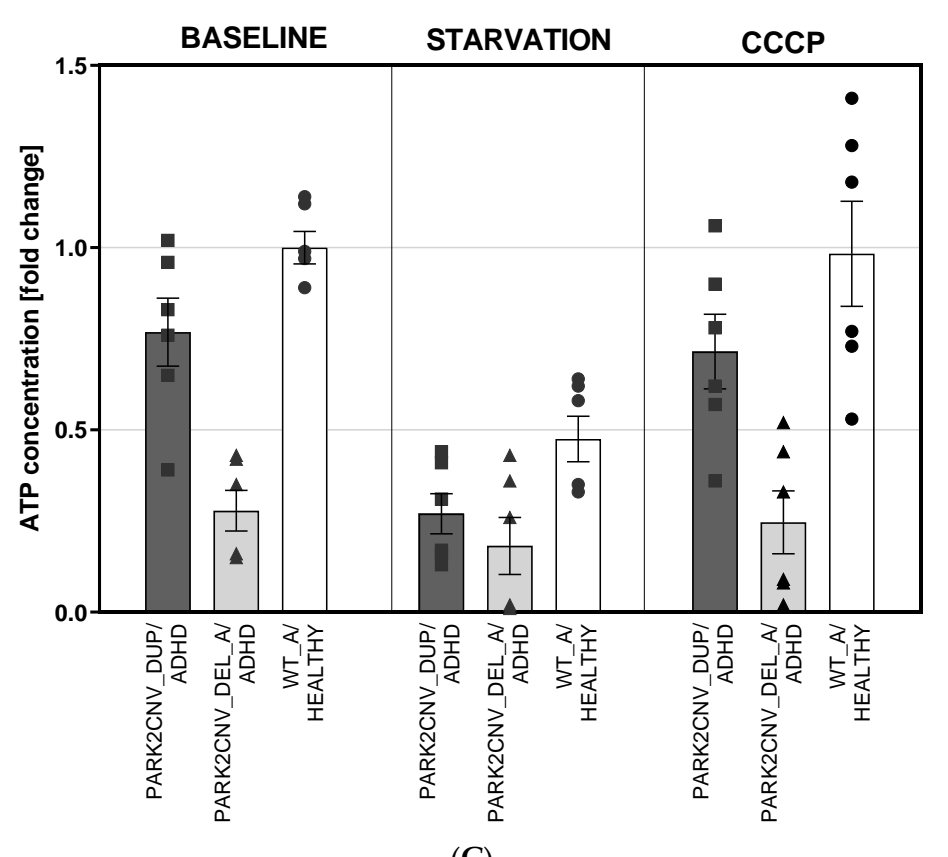

(C)

Figure 3. (A-C): ATP quantification. Cellular ATP content was evaluated in HDF lines $(\mathbf{A}, \mathbf{B})$ and in mDANs (C) in baseline conditions, after 24-h starvation stress and after treatment with $10 \mu \mathrm{M} \mathrm{CCCP}$ for $24 \mathrm{~h}$. Fold difference was calculated against WT in baseline conditions. Data were obtained from two independent experiments with samples measured in triplicate. Data are shown as mean \pm SEM. Exploratory ANOVA was calculated. Figure A shows the data of HDF cell lines from PARK2 CNV duplication vs. deletion vs. wildtype healthy control separately, Figure B shows a combined analysis of PARK2 CNV deletion + duplication vs. wildtype ADHD + healthy to increase number of HDF cell lines and statistical power. Level of significance was set at $p=0.05 .{ }^{*} p \leq 0.05 ;{ }^{* *} p \leq 0.01 ;{ }^{* * *} p \leq 0.001$.

The mDANs showed lower levels of ATP from cultures subjected to starvation compared to baseline conditions (Tukey HSD $p<0.0001$, Figure 3B) whereas the CCCP treatment seems to have a lesser effect on mDANs. Genotype was found to have a significant effect on ATP content, and decreased ATP levels were observed in both PARK2 CNV deletion (Tukey HSD $p<0.0001$ ) and PARK2 CNV duplication/ADHD compared to WT healthy control (Tukey HSD $p=0.004$ ) (see Figure 3C).

\subsection{Oxygen Consumption Rates (OCR)}

We measured the basal extracellular oxygen consumption rate (OCR) in the HDF and mDANs under baseline conditions, 24-h starvation stress, and 24-h CCCP treatment (Figure 4A-F and Figure 5A-C) and additional dimension of energy metabolism. Because the PARK2 duplication as well as the deletion carriers showed an effect in the same direction, we analyzed the data from the HDF first separately and then together to increase the statistical power. The time course recording was arbitrarily divided into three bins of 30 min each $\left(\mathrm{I}_{0-30}, \mathrm{I}_{30-60}, \mathrm{I}_{60-90}\right)$ to avoid multiple testing and because repeated measured ANOVA was technically not possible to conduct with all the measured time points. Student's tests were run for each interval to determine if there were differences between the genotypes in baseline OCR. Statistical analysis showed significant differences between genotypes both under baseline conditions (Figure 4A,D) and after starvation (Figure 4B,E). HDF of PARK2 CNVs carriers revealed lower rates of extracellular oxygen consumptions in comparison to WT under baseline conditions (see Figure 4A) and after starvation (Figure 4B,E). We could not detect a significant difference between PARK2 CNV deletion and duplication carriers and WT healthy and ADHD control after CCCP treatment (see Figure 4C,F). Our data therefore suggest that HDF derived from ADHD patients carrying PARK2 CNV deletion and duplication display decreased extracellular oxygen consumption compared to WT under both baseline conditions and after starvation stress, but not after CCCP treatment. 


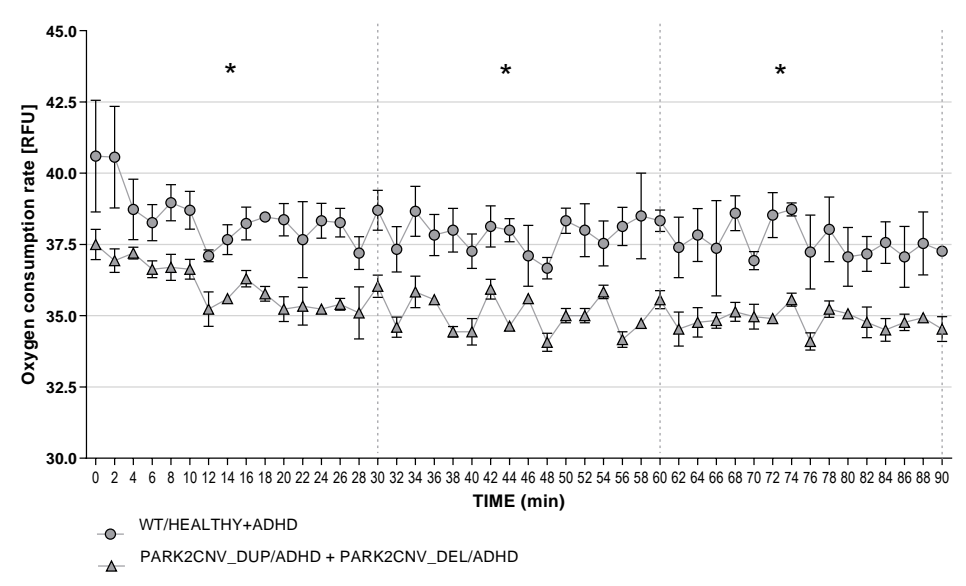

(A)

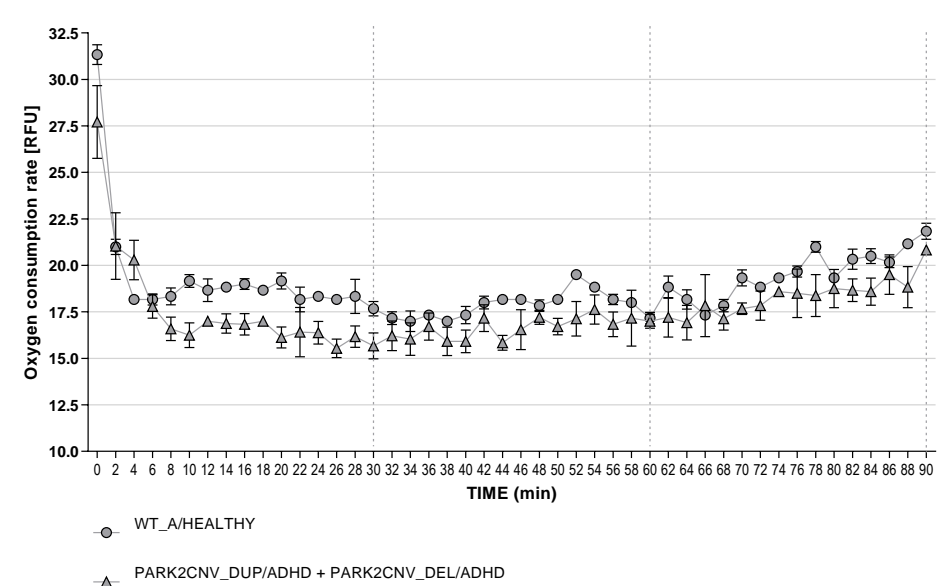

(C)

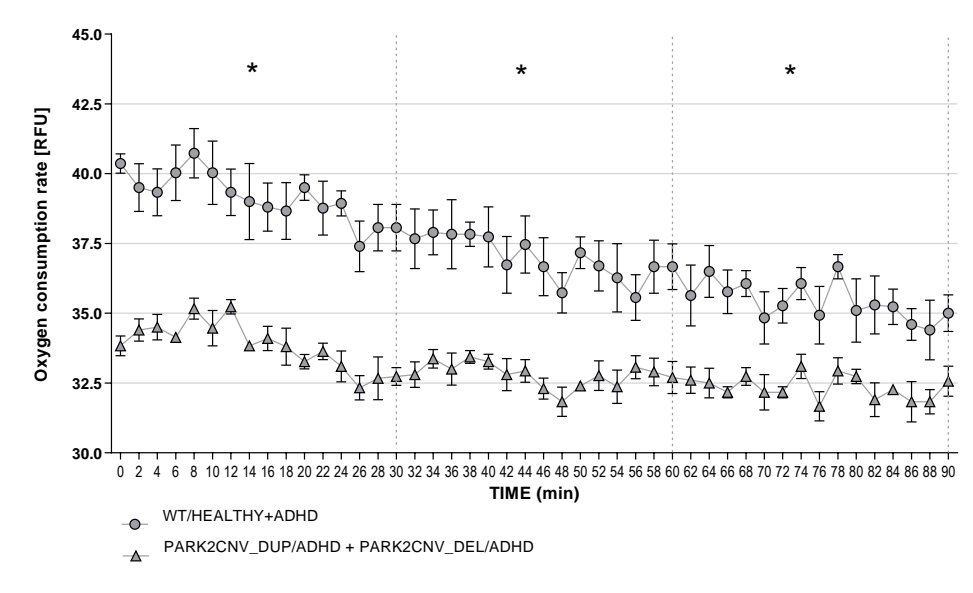

(B)

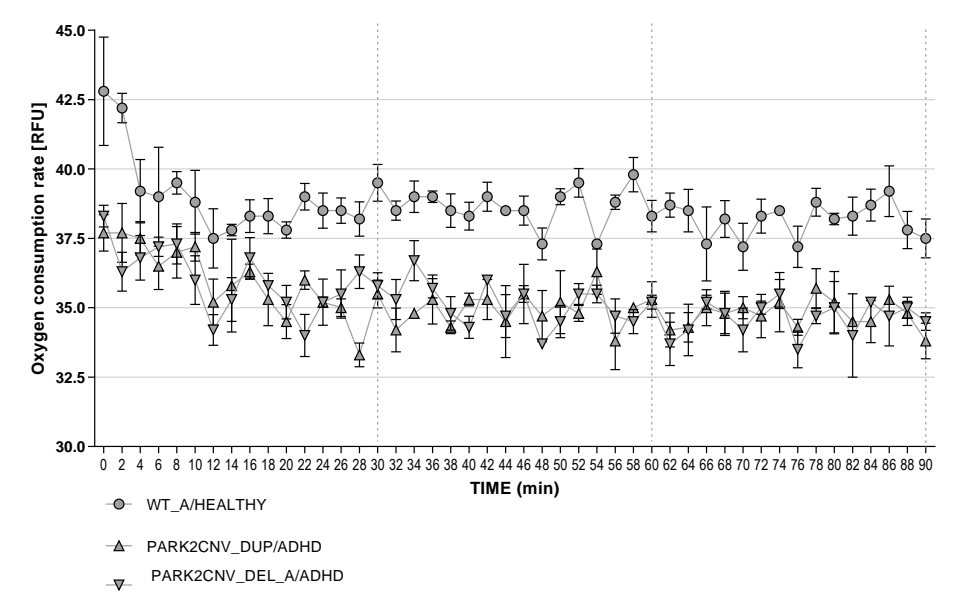

(D)

Figure 4. Cont. 


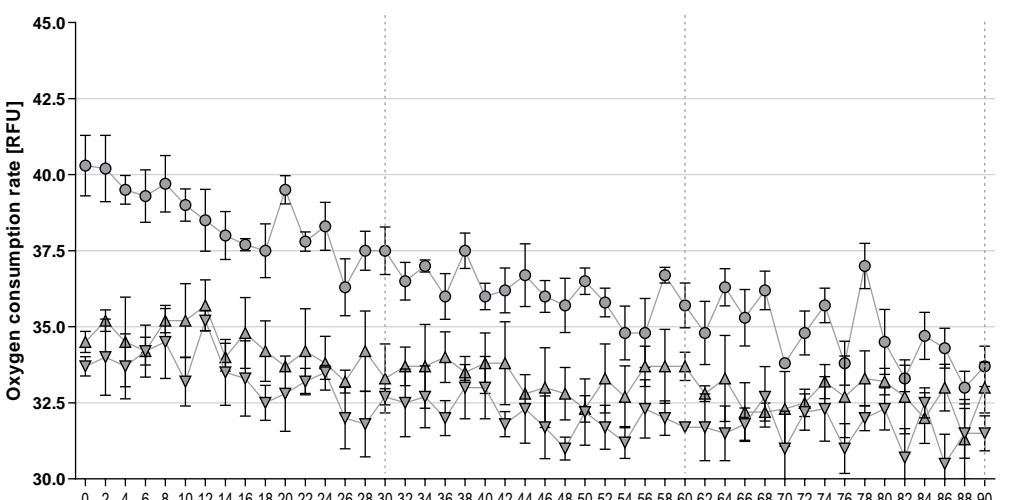

- WT_AREALTHY

$\triangle$ PARK2CNV_DUP/ADHD

7. PARK2CNV_DEL_A/ADHD
TIME (min)

(E)



(F)

Figure 4. (A-F): Oxygen consumption rate in human-derived fibroblasts (HDF). Basal oxygen consumption was measured under baseline (A,D), starvation (B,E), and CCCP treatment conditions (C,F). Fluorescence (RFU-y-axis) signal correlating with the respiration was recorded every two minutes for 90 min. Data are presented as mean of two independent experiments with samples measured in triplicate. Figure 4A-C show a combined analysis of PARK2 CNV deletion + duplication vs. wildtype healthy+ADHD wildtype to increase number of HDF cell lines and statistical power). HDF derived from ADHD patients carrying PARK2 CNVs showed lower rates of extracellular oxygen consumptions compared to WT in all the intervals both under baseline conditions $\left(\mathrm{I}_{0-30}\right.$ : $\mathrm{CNV}$ carriers $(\mathrm{M}=36.056$; $\mathrm{SD}=0.106)$, $\mathrm{WT}(\mathrm{M}=38.493 ; \mathrm{SD}=1.276) ; \mathrm{I}_{30-60}$ : CNV carriers $(\mathrm{M}=35.026 ; \mathrm{SD}=0.068), \mathrm{WT}(\mathrm{M}=37.859 ; \mathrm{SD}=1.209) ; \mathrm{I}_{60-90}: \mathrm{CNV}$ carriers $(\mathrm{M}=34.841 ; \mathrm{SD}=0.293), \mathrm{WT}(\mathrm{M}=37.626$; $\mathrm{SD}=1.279)$ (see Figure $4 \mathrm{~A})$ and after starvation $\left(\mathrm{I}_{0-30}: \mathrm{CNV}\right.$ carriers $(\mathrm{M}=34.938 ; \mathrm{SD}=1.267)$, $\mathrm{WT}(\mathrm{M}=39.160 ; \mathrm{SD}=1.398) ; \mathrm{I}_{30-60}$ : $\mathrm{CNV}$ carriers $(\mathrm{M}=33.907$; $\mathrm{SD}=1.296)$, $\mathrm{WT}(\mathrm{M}=36.970 ; \mathrm{SD}=1.527) ; \mathrm{I}_{60-90}$ : $\mathrm{CNV}$ carriers $(\mathrm{M}=33.593 ; \mathrm{SD}=1.427), \mathrm{WT}(\mathrm{M}=35.430 ; \mathrm{SD}=1.265)$. Figure $4 \mathrm{D}-\mathrm{F}$ shows the data of HDF cell lines from PARK2 CNV duplication vs. deletion vs. wildtype healthy control separately. There was a significant difference between the genotypes in all intervals in baseline: $\mathrm{I}_{0-30}: \mathrm{t}_{(4)}=3.292, p=0.030, d=2.692, r=0.803 ; \mathrm{I}_{30-60}: \mathrm{t}_{(4)}=4.054, p=0.015, d=3.310, r=0.856 ; \mathrm{I}_{60-90}: \mathrm{t}_{(4)}=3.675, p=0.0321, d=3.001, r=0.832$ and as well after starvation: starvation $\mathrm{I}_{0-30}: \mathrm{t}_{(4)}=6.224, p=0.003, d=5.082, r=0.931 ; \mathrm{I}_{30-60}: \mathrm{t}_{(4)}=4.351, p=0.012, d=3.553, r=0.871 ; \mathrm{I}_{60-90}: \mathrm{t}_{(4)}=3.842, p=0.018, d=3.337, r=0.843$. Data are shown as \pm standard error of mean (SEM). Exploratory repeated measure ANOVAs and t-tests were calculated. Level of significance was set at $p=0.05$. ${ }^{*} p \leq 0.05$. 
To determine if with time the recorded fluorescence would decrease, we calculated an exploratory repeated measures ANOVA on RFU recorded in the intervals. In the baseline conditions, a significant effect of time $\left(\mathrm{F}_{(2,8)}=71.024, p<0.0001\right)$ could be seen, but no interaction between time and genotype. This suggests that the fluorescence signal declines over time, possibly because of the utilization of the reagent, which was unaffected by genotype. This finding was similar in the samples subjected to 24-h starvation $\left(\mathrm{F}_{(2,14)}=411,536, p<0.0001\right)$, but in this case the decline was higher in healthy and ADHD wildtype control lines, potentially because of the earlier reported elevated OCR compared to the PARK2 CNV deletion and duplication carriers $\left(\mathrm{F}_{(2,14)}=88.790, p<0.0001\right)$.

Because of only having $\mathrm{n}=1$ per group in the mDANs we could not perform valid statistical tests with biological replicates. Numerically, also in the mDANs, duplication as well as deletion carriers were observed to have a lower OCR under baseline conditions and, although to a lesser degree, after nutrient deprivation (see Figure 5A,B). After CCCP treatment, the mDANs from especially the ADHD PARK2 deletion carrier showed lower OCR in comparison to the Healthy WT control (see Figure 5C).

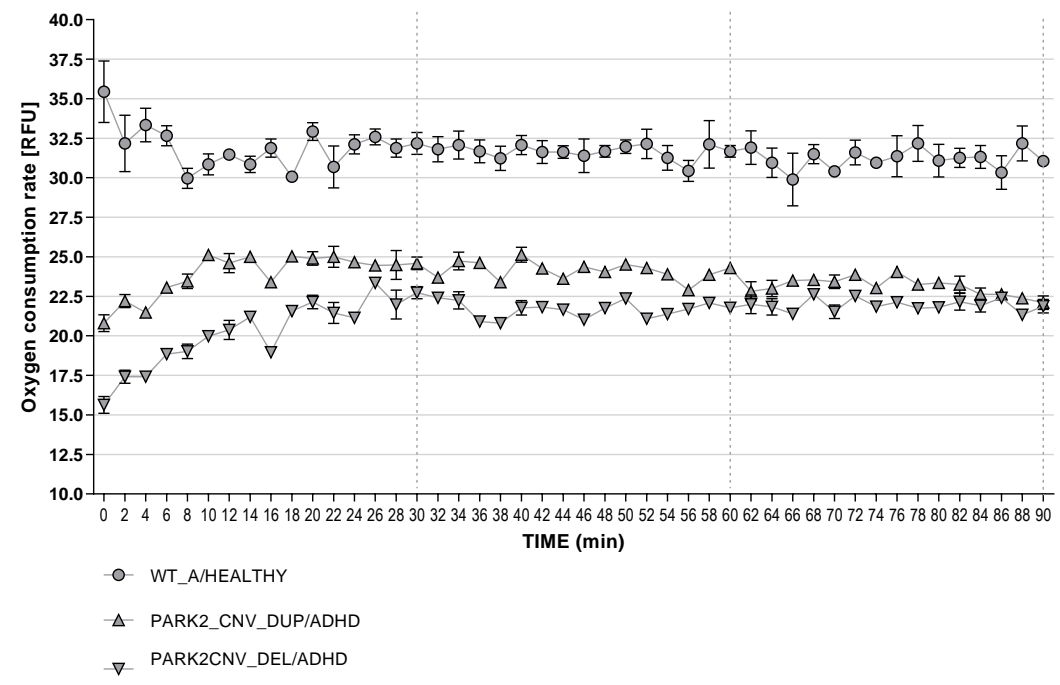

(A)

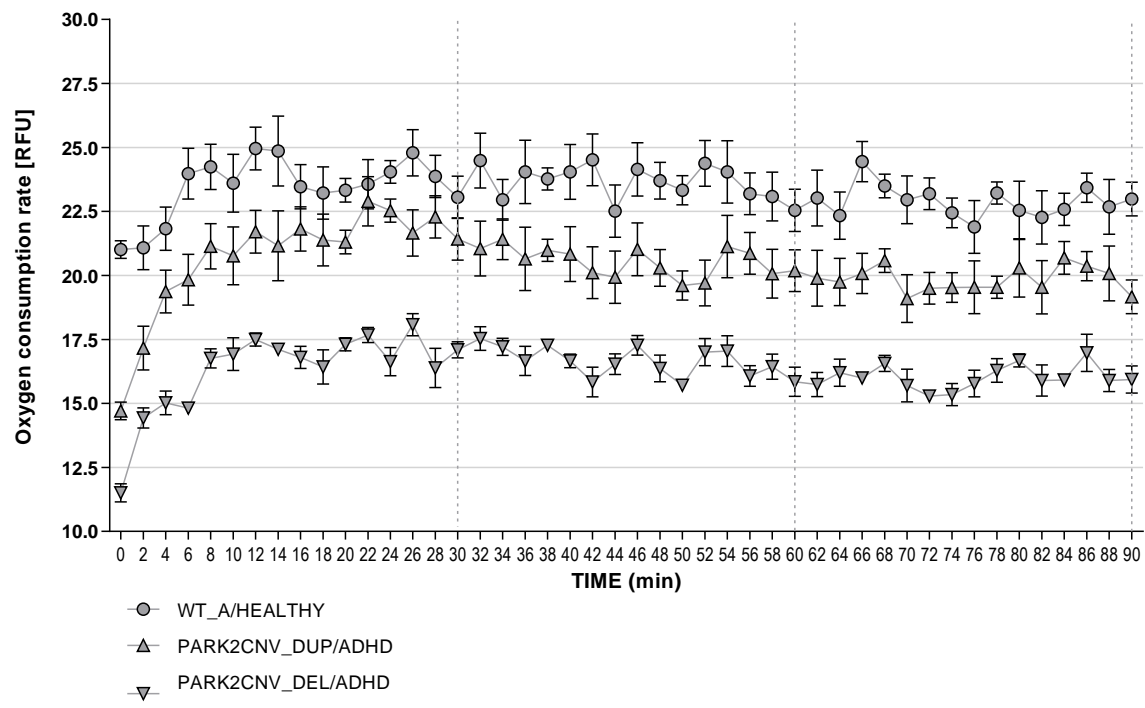

(B)

Figure 5. Cont. 


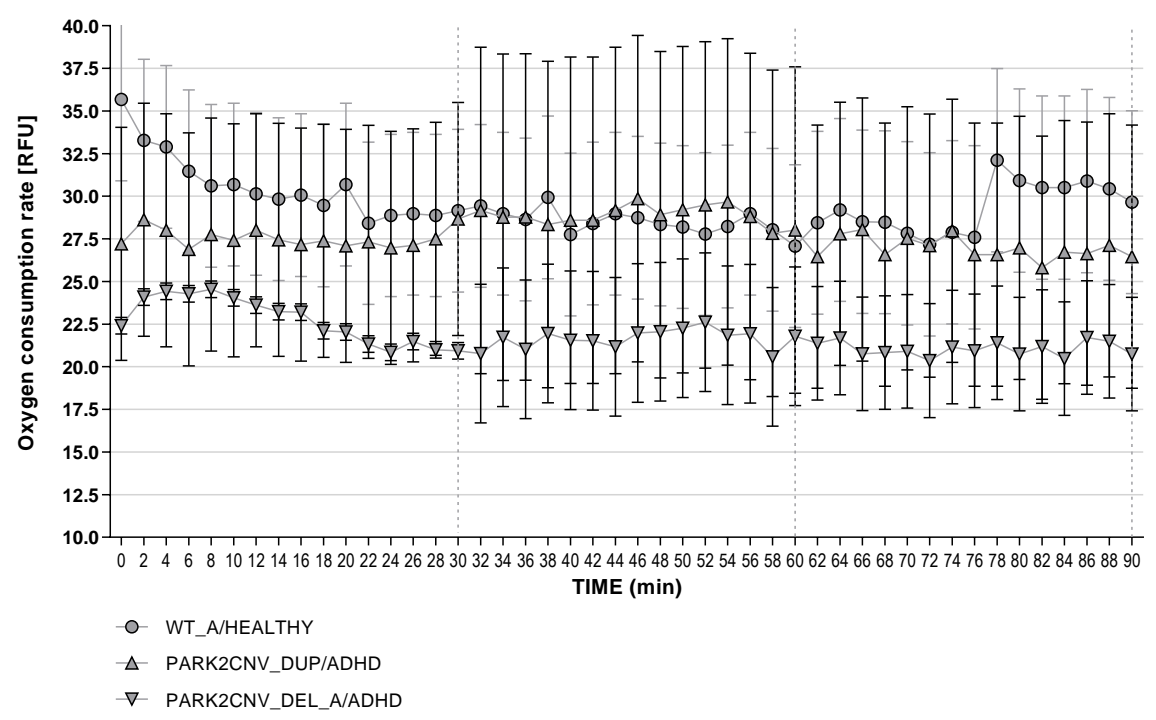

(C)

Figure 5. (A-C): Oxygen consumption rate in mDAN. Basal oxygen consumption rate was evaluated both under baseline (A), starvation (B), and CCCP treatment conditions (C). Fluorescence (RFU-y-axis) signal correlating with the respiration was recorded every two minutes for $90 \mathrm{~min}$. Data are presented as mean of two independent experiments with samples measured in triplicates. Because the biological replicates were $\mathrm{n}=1$ for each group, the data shown here are only descriptive.

\subsection{Reactive Oxygen Species Production (ROS)}

Finally, we analyzed the effect of the different genotypes on the production of cellular ROS in fibroblast (Figure S6A,C). ROS measurement showed a significant effect of the nutrient deprivation and CCCP stress $\left(\mathrm{F}_{(2,12)}=55.208, p<0.0001, \eta_{\mathrm{p}}{ }^{2}=0.902\right)$ but not of genotype $\left(\mathrm{F}_{(1,12)}=2.183, p=0.165\right.$, $\left.\eta_{\mathrm{p}}^{2}=0.154\right)$, and also no interaction effect $\left(\mathrm{F}_{(2,12)}=0.768, p=0.485, \eta_{\mathrm{p}}{ }^{2}=0.114\right)$. The main differences for treatment were found between baseline and starvation conditions (Tukey HSD $p<0.0001$ ), and baseline and CCCP treatment (Tukey HSD $p<0.0001$ ). A higher amount of ROS could be observed after starvation $(\mathrm{M}=2.441 ; \mathrm{SD}=0.262)$ and $\mathrm{CCCP}$ treatment $(\mathrm{M}=2.268 ; \mathrm{SD}=0.064)$ compared to baseline conditions $(\mathrm{M}=1.397 ; \mathrm{SD}=0.184)$. We did not find a significant difference in ROS response between starvation and CCCP treatment (Tukey HSD $p=0.274$ ). In the mDANs, PARK2 CNV carriers seemed to display a lower amount of ROS in comparison to the healthy WT. Both starvation and CCCP treatment increased the ROS amount in all three mDAN cell lines (Figure S6B).

\section{Discussion}

In our study we investigated two patient-derived cellular models, HDF and also preliminary results from hiPSC-derived mDANs, obtained from adult ADHD patients. The patients we derived mDANs from were CNV carriers of deletions or duplications in the PARK2 gene, which has previously been associated with ADHD and were compared with healthy and ADHD wildtype carriers [15]. We used two stress paradigms, nutrient deprivation and CCCP treatment, to attempt to enhance the cellular pathophenotype in our experiments. The PARK2-coded protein parkin is involved in mitochondrial functions such as mitophagy, fusion and fission, biogenesis and mitochondrial transport [36]. Regarding PARK2 gene expression, we could find the lowest levels in the deletion carrier, however, also the duplication carrier had lower PARK2 gene as well as protein concentration in comparison to the wildtypes. The molecular mechanism of how the duplication might lead to decreased PARK2 gene and protein expression needs to be explored in future studies. In HDF, as well nutrient deprivation as CCCP treatment led to decreased PARK2 protein concentrations in all cell lines independent from the genotype. In mDAN this was true for the WT healthy control cell line, but not for the CNV cell lines. However, because of the preliminary and exploratory 
data on the mDAN, no definite conclusion can be drawn from those findings. We also assessed the morphology of mitochondria in fibroblasts from ADHD PARK2 CNV carriers in comparison to wildtype carriers. An altered mitochondrial network morphology was observed in the PARK2 duplication carrier/ADHD. Starvation stress affected all of our fibroblasts independently of genotype and led to a more fragmented mitochondrial network branching with single elements of a rather spherical shape. On a descriptive level, the PARK2 CNV duplication carrier/ADHD under baseline conditions seemed to show a more elongated mitochondrial shape and increased tubular branching compared to PARK2 CNV deletion carriers/ADHD and WT control and ADHD. Similar alterations were reported in cells derived from Parkinson's patients carrying mutations in the PARK2 gene, with both increased tubular branching [28] and fragmented structure [37] reported. Additionally, in high-aged Park2 knockout mice, more fragmented mitochondria were observed in conjunction with locomotor impairments [38]. Taken together, the data suggests that genetic variants and mutations in PARK2 might result in protein dysfunction, thereby impacting mitochondrial stability. ADHD and ASD are neurodevelopmental and not neurodegenerative disorders, however, genetic variation in PARK2 gene and environmental stressor might lead to impairment of mitochondrial structure in the neurodevelopment even though no further neurodegeneration appears later in life.

In the past years, several studies have linked mitochondrial dysfunction to the etiology of several mental disorders [39]. The brain is an organ that is in need of high energy supply, and it is well established that mitochondria play a crucial role in the metabolism of not only energy, but also amino acids, lipids, and steroids; all of which are essential elements for a normally functioning central nervous system [40]. Moreover, specifically in synapses, mitochondria contribute to the maintenance of the membrane potential, facilitate calcium-dependent neurotransmitter release, and activate second messenger pathways [41,42]. After observing altered mitochondrial structure in our HDFs, we investigated mitochondria-related energy metabolism by measuring ATP levels and oxygen consumption rate in HDF and mDANs, under baseline and two stress paradigm conditions. Our findings indicate a significant difference between genotypes in HDF under both baseline and starvation conditions, and a similar result was observed in mDANs which however needs to be evaluated in the future with an independent set of additional cell lines. ADHD PARK2 CNV duplication and deletion carriers showed lower levels of cellular ATP compared to healthy and ADHD wildtype controls, suggesting a potential dysfunctional MQC system. This may contribute to an aggregation of damaged mitochondria and ATP loss. This finding is supported by a previous study using fibroblast of carriers of different PARK2 gene mutations. Under baseline conditions, ADHD PARK2 CNV deletion and duplication carriers also demonstrated decreased extracellular oxygen consumption rates under both baseline and starvation conditions in HDF, which was again reflected in the mDANs. These results replicate previous findings using ADHD cybrid cells; a transgenic cell model that allows the effects of a patient's mitochondria to be studied in isolation. This study reported decreased levels of ATP production and oxygen consumption, and higher levels of superoxide radicals [21]. CCCP treatment had no effect on ATP levels or oxygen consumption rate in HDF or mDANs, apart from reduced OCR in ADHD PARK2 CNV deletion carrier mDANs. This finding was unexpected, as CCCP has been shown to cause mitochondrial depolarization in several other studies. However, it could be that the concentrations used in our study were too low [43].

The physiological function of mitochondria leads to the formation of several reactive oxygen species (ROS) and reactive nitrogen species (RNS), which under normal conditions are attenuated by the redox scavenger system [44]. We therefore aimed at evaluating whether the concentration of ROS was different because of genotype and/or stress paradigms. As expected, our data suggested that the cells had increased ROS production under starvation and CCCP treatment compared to baseline. However, there were no genotypic differences regarding ROS levels.

Our results, at least in part, could reflect dysregulated mitophagy (via PARK2/PRKN and PINK1). It has been shown that parkin is transported to the mitochondria which have an impaired energy metabolism, where it ubiquitinates mitofusin (Mfn) to mark them for degradation and 
mitophagy [45]. If parkin is not functioning properly, impaired mitochondria can accumulate and lead to neurodegeneration [46]. As no neurodegeneration is described in ADHD and ASD, any genetically induced Parkin alterations might have a more subtle effect, which leads to a higher rate of sub-optimal functioning mitochondria. This is supported by our findings of lower ATP content and reduced oxygen consumption rate, but not to an accumulation of defect mitochondria in the cells, at least not in an amount that would cause neurodegeneration. However, we could also show evidence for a stronger vulnerability of the ADHD PARK2 CNV deletion and duplication carrier cells to nutrient deprivation stress which has also been described in other Parkin-or PINK1-deficient models [43,47].

The regulation of oxidative stress, measured by the level of ROS, was not altered in our ADHD PARK2 CNV deletion or duplication cells, suggesting there could be alternative compensatory pathways regarding mitochondrial or metabolic stress. Recent studies give evidence of oxidative and nitrosative stress markers being altered in ADHD compared to healthy controls, as reviewed by Lopresti and colleagues [48]. Despite the results of those studies hinting at higher oxidative stress and less sufficient response to oxidative damage both in children and adults with ADHD [49], reported findings are partially contradictory. This is most likely due to inconsistences in markers tested, publication bias, sample collection, and the population studied [48]. However, we investigated ADHD patients that are carriers of a rare genetic variant, so our results cannot be generalized for a majority of ADHD patients.

\section{Conclusions}

Our work suggests that ADHD PARK2 CNV carriers might have an energy impairment. This impairment could be due to the diverse role of PARK2 in mitochondrial dynamics, potentially leading to the disruption of normal brain plasticity and cellular resilience [39]. Several highly sensitive temporal windows, such as nervous system development during embryogenesis, could be more susceptible to such perturbations possibly leading to a role in the etiology of neurodevelopmental diseases. During this sensitive developmental stage, mitochondria are involved in the maturation of neural stem cells, proliferation and differentiation, formation of dendritic processes, and synaptic plasticity [50-53]. PARK2 and PINK1 are the main regulators of the MQC, and thus could play a functional role in psychiatric and neurodevelopmental disorders like subgroups of patients with ADHD. Therefore, as novel therapeutic options, substances that increase mitochondrial functions and decrease oxidative stress like for example antioxidants (e.g., polyphenols) should be investigated in preclinical and clinical studies for their efficacy as (add-on) treatment of ADHD.

\subsection{Limitations}

The results of our study should be considered under several limitations. First, the number of biological repeats (patients and controls) was low, especially in the mDAN, and we did not use isogenic controls. Second, although the models presented in this study are one of the few available cellular models for studying ADHD, PARK2 CNVs represent a rare variation found just in a small subset of ADHD patients. Furthermore, we did not conduct electrophysiological experiments yet to investigate basal neuronal functions besides the ability to produce dopamine. Additionally, we did not in detail investigate the $\mathrm{CNV}$ effect on neuronal function and survival which will be done in future studies.

\subsection{Data Availability}

The data that support these findings are available from the corresponding author, S.K.-S, upon reasonable request. Human subject data will be deidentified to protect confidentiality.

Supplementary Materials: The following are available online at http://www.mdpi.com/2077-0383/9/12/4092/s1, Figure S1: Pluripotency tests and markers, Figure S2: Dopaminergic markers of differentiated neuronal cells, Figure S3: PARK2 gene locus and relatedness matrix of DNA from generated hiPSC cells and fibroblasts of PARK2 CNV carrier, Figure S4: Copy number variation plot of all generated hiPSC lines, Figure S5: Chromosome 6 ideogram and SNP array analysis of the PARK2 locus, Figure S6: ROS production assay, Table S1: Phenotypic and demographic data, Table S2: Primer sequences for PCR and qRT PCR, Table S3: Primary and secondary antibodies 
used for immunocytochemistry, Table S4: PARK 2 gene expression in HDF, Supplementary Materials 1: Material and Methods Details.

Author Contributions: Conceptualization, A.R. and S.K.-S.; methodology, V.S.P.; K.G., F.E., D.H., O.B.; A.T., S.H., S.K.-S.; software, V.S.P., A.G.C.; validation, L.F., V.S.P.; formal analysis, S.K.-S., V.S.P.; investigation, V.S.P., L.F., S.H., A.T., P.H., S.K.-S., D.H., R.M.; resources, O.B., F.E., K.G.; data curation, A.C.G., D.H.; writing-original draft preparation, V.S.P., S.K.-S.; writing—review and editing, F.R., R.M., O.B., A.T., A.G.C., A.R., D.H., L.F., K.G., F.E.; visualization, V.S.P., S.K.-S.; supervision, S.K.-S., A.R., O.B., F.E.; project administration, S.K.-S.; funding acquisition, A.R., O.B., S.K.-S. All authors have read and agreed to the published version of the manuscript.

Funding: This study was supported from the European Union's Horizon 2020 research and innovation program under the Marie Sklodowska-Curie grant agreement No 643051 (to A.R.), the European Regional Development Fund (Stem Cell Factory III; grant EFRE-0800978, to O.B.), the H2020 Consortium "NSC-Reconstruct" (grant agreement No. 874758 , to O.B.) and the Young Researcher Program of the University Hospital Frankfurt as well as the young researcher funding from the IZKF Würzburg (Z3-24 to S.K.-S.). This publication was supported by the Open Access Publication Fund of the University of Würzburg. This work has been supported by the ECNP Thematic Working Group 'iPSCs Platform for Neuropsychiatry.

Acknowledgments: We thank Joyce Auer, Sandra Winkler, Michaela Segschneider, and Theresia Töpner for the technical support and all the patients and controls who participated in this study.

Conflicts of Interest: S.K.S. has received travel grants and author's honoraria from Shire/Takeda and Medice Arzneimittel Pütter GmbH. A.R. has received travel grants and author's honoraria from Shire/Takeda and Medice Arzneimittel Pütter GmbH. All other authors do not declare a conflict of interest.

\section{Abbreviations}

ADHD
ASD
ATP
CCCP
CNV
HDF
hiPSC
mDAN
MQC
OCR
PARK2CNV_DUP/ADHD
PARK2CNV_DEL_A/ADHD
PARK2CNV_DEL_B/ADHD
PD NMS
ROS
UPDRS
WT/ADHD
WT_A/HEALTHY
WT_B/HEALTHY

Attention-Deficit-/Hyperactivity Disorder

Autism Spectrum Disorder

Adenosine Triphosphate

Carbonyl Cyanide m-Chlorophenyl Hydrazine

Copy Number Variation/Variants

Human Dermal Fibroblast

human induced Pluripotent Stem Cells

mature Dopaminergic Neurons

Mitochondria Quality Control system

Oxygen Consumption Rate

PARK2 duplication carrier with ADHD

PARK2 deletion carrier with ADHD, A

PARK2 deletion carrier with ADHD, B

Non-Motor Symptom Assessment Scale for Parkinson's disease

Reactive Oxygen Species

Unified Parkinson's Disease Rating Scale

Wildtype in PARK2 locus and ADHD

Wildtype in PARK2 locus and healthy control A

Wildtype in PARK2 locus and healthy control B

\section{References}

1. Bernardi, S.; Faraone, S.V.; Cortese, S.; Kerridge, B.T.; Pallanti, S.; Wang, S.; Blanco, C. The lifetime impact of attention deficit hyperactivity disorder: Results from the National Epidemiologic Survey on Alcohol and Related Conditions (NESARC). Psychol. Med. 2012, 42, 875-887. [CrossRef]

2. Farley, M.; Cottle, K.J.; Bilder, D.; Viskochil, J.; Coon, H.; McMahon, W. Mid-life social outcomes for a population-based sample of adults with ASD. Autism. Res. 2018, 11, 142-152. [CrossRef] [PubMed]

3. Baird, G.; Simonoff, E.; Pickles, A.; Chandler, S.; Loucas, T.; Meldrum, D.; Charman, T. Prevalence of disorders of the autism spectrum in a population cohort of children in South Thames: The Special Needs and Autism Project (SNAP). Lancet 2006, 368, 210-215. [CrossRef]

4. Brikell, I.; Kuja-Halkola, R.; Larsson, H. Heritability of attention-deficit hyperactivity disorder in adults. Am. J. Med. Genet. B Neuropsychiatr Genet. 2015, 168, 406-413. [CrossRef] [PubMed]

5. Waye, M.M.Y.; Cheng, H.Y. Genetics and epigenetics of autism: A Review. Psychiatry Clin. Neurosci. 2018, 72, 228-244. [CrossRef] [PubMed] 
6. Faraone, S.V.; Perlis, R.H.; Doyle, A.E.; Smoller, J.W.; Goralnick, J.J.; Holmgren, M.A.; Sklar, P. Molecular genetics of attention-deficit/hyperactivity disorder. Biol. Psychiatry 2005, 57, 1313-1323. [CrossRef] [PubMed]

7. Banerjee, T.D.; Middleton, F.; Faraone, S.V. Environmental risk factors for attention-deficit hyperactivity disorder. Acta Paediatr. 2007, 96, 1269-1274. [CrossRef]

8. Franke, B.; Michelini, G.; Asherson, P.; Banaschewski, T.; Bilbow, A.; Buitelaar, J.K.; Cormand, B.; Faraone, S.V.; Ginsberg, Y.; Haavik, J.; et al. Live fast, die young? A review on the developmental trajectories of ADHD across the lifespan. Eur. Neuropsychopharmacol. 2018, 28, 1059-1088.

9. Nikolas, M.A.; Burt, S.A. Genetic and environmental influences on ADHD symptom dimensions of inattention and hyperactivity: A meta-analysis. J. Abnorm. Psychol. 2010, 119, 1-17. [CrossRef]

10. Stankiewicz, Z.; Lupski, J.R. Structural variation in the human genome and its role in disease. Annu. Rev. Med. 2010, 61, 437-455. [CrossRef]

11. Elia, J.; Gai, X.; Xie, H.; Perin, J.; Geiger, E.; Glessner, J.; D’Arcy, M.; DeBerardinis, R.; Frackelton, E.; Kim, C.; et al. Rare structural variants found in attention-deficit hyperactivity disorder are preferentially associated with neurodevelopmental genes. Mol. Psychiatry 2010, 15, 637-646. [CrossRef] [PubMed]

12. Elia, J.; Glessner, J.T.; Wang, K.; Takahashi, N.; Shtir, C.J.; Hadley, D.; A Sleiman, P.M.; Zhang, H.; E Kim, C.; Robison, R.J.; et al. Genome-wide copy number variation study associates metabotropic glutamate receptor gene networks with attention deficit hyperactivity disorder. Nat. Genet. 2012, 44, 78-84. [CrossRef] [PubMed]

13. Jacob, C.P.; Weber, H.; Retz, W.; Kittel-Schneider, S.; Heupel, J.; Renner, T.; Lesch, K.-P.; Reif, A. Acetylcholine-metabolizing butyrylcholinesterase (BCHE) copy number and single nucleotide polymorphisms and their role in attention-deficit/hyperactivity syndrome. J. Psychiatr. Res. 2013, 47, 1902-1908. [CrossRef] [PubMed]

14. Martin, J.; Odonovan, M.C.; Thapar, A.; Langley, K.; Williams, N. The relative contribution of common and rare genetic variants to ADHD. Transl. Psychiatry 2015, 5, e506. [CrossRef] [PubMed]

15. Jarick, I.; Volckmar, A.-L.; Pütter, C.; Pechlivanis, S.; Nguyen, T.T.; Dauvermann, M.R.; Beck, S.; Albayrak, Ö.; Scherag, S.; Gilsbach, S.; et al. Genome-wide analysis of rare copy number variations reveals PARK2 as a candidate gene for attention-deficit/hyperactivity disorder. Mol. Psychiatry 2014, 19, 115-121. [CrossRef] [PubMed]

16. Scarffe, L.A.; Stevens, D.A.; Dawson, V.L.; Dawson, T.M. Parkin and PINK1: Much more than mitophagy. Trends Neurosci. 2014, 37, 315-324. [CrossRef] [PubMed]

17. Hang, L.; Thundyil, J.; Lim, K.L. Mitochondrial dysfunction and Parkinson disease: A Parkin-AMPK alliance in neuroprotection. Ann. N. Y. Acad. Sci. 2015, 1350, 37-47. [CrossRef]

18. Pickrell, A.M.; Youle, R.J. The roles of PINK1, parkin, and mitochondrial fidelity in Parkinson's disease. Neuron 2015, 85, 257-273. [CrossRef]

19. Rizzuto, R.; De Stefani, D.; Raffaello, A.; Mammucari, C. Mitochondria as sensors and regulators of calcium signalling. Nat. Rev. Mol. Cell Biol. 2012, 13, 566-578. [CrossRef]

20. Marazziti, D.; Baroni, S.; Picchetti, M.; Landi, P.; Silvestri, S.; Vatteroni, E.; Dell'Osso, M.C. Psychiatric disorders and mitochondrial dysfunctions. Eur. Rev. Med. Pharmacol. Sci. 2012, 16, 270-275.

21. Verma, P.; Singh, A.; Nthenge-Ngumbau, D.N.; Rajamma, U.; Sinha, S.; Mukhopadhyay, K.; Mohanakumar, K.P. Attention deficit-hyperactivity disorder suffers from mitochondrial dysfunction. BBA Clin. 2016, 6, 153-158. [CrossRef] [PubMed]

22. Bogetofte, H.; Jensen, P.; Okarmus, J.; Schmidt, S.I.; Agger, M.; Ryding, M.; Nørregaard, P.; Fenger, C.; Zeng, X.; Graakjær, J.; et al. Perturbations in RhoA signalling cause altered migration and impaired neuritogenesis in human iPSC-derived neural cells with PARK2 mutation. Neurobiol. Dis. 2019, 132, 104581. [CrossRef] [PubMed]

23. Bogetofte, H.; Jensen, P.; Ryding, M.; Schmidt, S.I.; Okarmus, J.; Ritter, L.; Worm, C.S.; Hohnholt, M.C.; Azevedo, C.; Roybon, L.; et al. PARK2 Mutation Causes Metabolic Disturbances and Impaired Survival of Human iPSC-Derived Neurons. Front. Cell Neurosci. 2019, 13, 297. [CrossRef] [PubMed]

24. Klinkenberg, M.; Gispert, S.; Dominguez-Bautista, J.A.; Braun, I.; Auburger, G.; Jendrach, M. Restriction of trophic factors and nutrients induces PARKIN expression. Neurogenetics 2012, 13, 9-21. [CrossRef]

25. Yamano, K.; Matsuda, N.; Tanaka, K. The ubiquitin signal and autophagy: An orchestrated dance leading to mitochondrial degradation. EMBO Rep. 2016, 17, 300-316. [CrossRef]

26. Lehrl, S.; Triebig, G.; Fischer, B. Multiple choice vocabulary test MWT as a valid and short test to estimate premorbid intelligence. Acta Neurol. Scand 1995, 91, 335-345. [CrossRef] 
27. Reif, A.; Herterich, S.; Strobel, A.; Ehlis, A.-C.; Saur, D.; Jacob, C.P.; Wienker, T.; Töpner, T.; Fritzen, S.; Walter, U.; et al. A neuronal nitric oxide synthase (NOS-I) haplotype associated with schizophrenia modifies prefrontal cortex function. Mol. Psychiatry 2006, 11, 286-300. [CrossRef]

28. Zanellati, M.C.; Monti, V.; Barzaghi, C.; Reale, C.; Nardocci, N.; Albanese, A.; Valente, E.M.; Ghezzi, D.; Garavaglia, B. Mitochondrial dysfunction in Parkinson disease: Evidence in mutant PARK2 fibroblasts. Front. Genet. 2015, 6, 78. [CrossRef]

29. Chu, C.-L.; Lee, I.H.; Chi, M.H.; Chen, K.C.; Chen, P.S.; Yao, W.J.; Jou, I.-M.; Yang, Y.K. Availability of dopamine transporters and auditory P300 abnormalities in adults with attention-deficit hyperactivity disorder: Preliminary results. CNS Spectr. 2018, 23, 264-270. [CrossRef]

30. Challman, T.D.; Lipsky, J.J. Methylphenidate: Its pharmacology and uses. Mayo Clin. Proc. 2000, 75, 711-721. [CrossRef]

31. Kriks, S.; Shim, J.-W.; Piao, J.; Ganat, Y.M.; Wakeman, D.R.; Xie, Z.; Carrillo-Reid, L.; Auyeung, G.; Antonacci, C.; Buch, A.; et al. Dopamine neurons derived from human ES cells efficiently engraft in animal models of Parkinson's disease. Nature 2011, 480, 547-551. [CrossRef] [PubMed]

32. Weykopf, B.; Haupt, S.; Jungverdorben, J.; Flitsch, L.J.; Hebisch, M.; Liu, G.H.; Suzuki, K.; Belmonte, J.C.I.; Peitz, M.; Blaess, S.; et al. Induced pluripotent stem cell-based modeling of mutant LRRK2-associated Parkinson's disease. Eur. J. Neurosci. 2019, 49, 561-589. [CrossRef] [PubMed]

33. Burbulla, L.F.; Kruger, R. The use of primary human fibroblasts for monitoring mitochondrial phenotypes in the field of Parkinson's disease. J. Vis. Exp. 2012, 68, e4228. [CrossRef] [PubMed]

34. Pickrell, A.M.; Huang, C.-H.; Kennedy, S.R.; Ordureau, A.; Sideris, D.P.; Hoekstra, J.G.; Harper, J.W.; Youle, R.J. Endogenous Parkin Preserves Dopaminergic Substantia Nigral Neurons following Mitochondrial DNA Mutagenic Stress. Neuron 2015, 87, 371-381. [CrossRef] [PubMed]

35. Flinn, L.; Mortiboys, H.; Volkmann, K.; Köster, R.W.; Ingham, P.W.; Bandmann, O. Complex I deficiency and dopaminergic neuronal cell loss in parkin-deficient zebrafish (Danio rerio). Brain 2009, 132, 1613-1623. [CrossRef] [PubMed]

36. Sarraf, S.A.; Raman, M.; Guarani-Pereira, V.; Sowa, M.E.; Huttlin, E.L.; Gygi, S.P.; Harper, J.W. Landscape of the PARKIN-dependent ubiquitylome in response to mitochondrial depolarization. Nature 2013, 496, 372-376. [CrossRef]

37. Haylett, W.; Swart, C.; Van Der Westhuizen, F.; Van Dyk, H.; Van Der Merwe, L.; Van Der Merwe, C.; Loos, B.; Carr, J.; Kinnear, C.; Bardien, S. Altered Mitochondrial Respiration and Other Features of Mitochondrial Function in Parkin-Mutant Fibroblasts from Parkinson's Disease Patients. Parkinsons Dis. 2016, 2016, 1819209.

38. Noda, S.; Sato, S.; Fukuda, T.; Tada, N.; Uchiyama, Y.; Tanaka, K.; Hattori, N. Loss of Parkin contributes to mitochondrial turnover and dopaminergic neuronal loss in aged mice. Neurobiol. Dis. 2020, 136, 104717. [CrossRef]

39. McCann, R.F.; Ross, D.A. So Happy Together: The Storied Marriage Between Mitochondria and the Mind. Biol. Psychiatry 2018, 83, e47-e49. [CrossRef]

40. Manji, H.K.; Kato, T.; Di Prospero, N.A.; Ness, S.; Beal, M.F.; Krams, M.; Chen, G. Impaired mitochondrial function in psychiatric disorders. Nat. Rev. Neurosci. 2012, 13, 293-307. [CrossRef]

41. Sheng, Z.H.; Cai, Q. Mitochondrial transport in neurons: Impact on synaptic homeostasis and neurodegeneration. Nat. Rev. Neurosci. 2012, 13, 77-93. [CrossRef] [PubMed]

42. Srivastava, R.; Faust, T.; Ramos, A.; Ishizuka, K.; Sawa, A. Dynamic Changes of the Mitochondria in Psychiatric Illnesses: New Mechanistic Insights From Human Neuronal Models. Biol. Psychiatry 2018, 83, 751-760. [CrossRef] [PubMed]

43. Bonello, F.; Hassoun, S.-M.; Mouton-Liger, F.; Shin, Y.S.; Muscat, A.; Tesson, C.; Lesage, S.; Beart, P.M.; Brice, A.; Krupp, J.; et al. LRRK2 impairs PINK1/Parkin-dependent mitophagy via its kinase activity: Pathologic insights into Parkinson's disease. Hum. Mol. Genet. 2019, 28, 1645-1660. [CrossRef] [PubMed]

44. Wang, W.; Fang, H.; Groom, L.; Cheng, A.; Zhang, W.; Liu, J.; Wang, X.; Li, K.; Han, P.; Zheng, M.; et al. Superoxide flashes in single mitochondria. Cell 2008, 134, 279-290. [CrossRef] [PubMed]

45. Ziviani, E.; Whitworth, A.J. How could Parkin-mediated ubiquitination of mitofusin promote mitophagy? Autophagy 2010, 6, 660-662. [CrossRef] [PubMed]

46. Gai, W.P.; Yuan, H.; Li, X.; Power, J.; Blumbergs, P.; Jensen, P. In situ and in vitro study of colocalization and segregation of alpha-synuclein, ubiquitin, and lipids in Lewy bodies. Exp. Neurol. 2000, 166, 324-333. [CrossRef] 
47. Gispert, S.; Ricciardi, F.; Kurz, A.; Azizov, M.; Hoepken, H.-H.; Becker, D.; Voos, W.; Leuner, K.; Müller, W.E.; Kudin, A.P.; et al. Parkinson phenotype in aged PINK1-deficient mice is accompanied by progressive mitochondrial dysfunction in absence of neurodegeneration. PLoS ONE 2009, 4, e5777. [CrossRef]

48. Lopresti, A.L. Oxidative and nitrosative stress in ADHD: Possible causes and the potential of antioxidant-targeted therapies. Atten. Defic. Hyperact. Disord. 2015, 7, 237-247. [CrossRef]

49. Joseph, N.; Zhang-James, Y.; Perl, A.; Faraone, S.V. Oxidative Stress and ADHD: A Meta-Analysis. J. Atten. Disord. 2015, 19, 915-924. [CrossRef]

50. Li, Z.; Okamoto, K.-I.; Hayashi, Y.; Sheng, M. The importance of dendritic mitochondria in the morphogenesis and plasticity of spines and synapses. Cell 2004, 119, 873-887. [CrossRef]

51. Mattson, M.P.; Gleichmann, M.; Cheng, A. Mitochondria in neuroplasticity and neurological disorders. Neuron 2008, 60, 748-766. [CrossRef] [PubMed]

52. Xavier, J.M.; Rodrigues, C.M.; Sola, S. Mitochondria: Major Regulators of Neural Development. Neuroscientist 2016, 22, 346-358. [CrossRef] [PubMed]

53. Cheng, N.; Rho, J.M.; Masino, S.A. Metabolic Dysfunction Underlying Autism Spectrum Disorder and Potential Treatment Approaches. Front. Mol. Neurosci. 2017, 10, 34. [CrossRef] [PubMed]

Publisher's Note: MDPI stays neutral with regard to jurisdictional claims in published maps and institutional affiliations.

(C) 2020 by the authors. Licensee MDPI, Basel, Switzerland. This article is an open access article distributed under the terms and conditions of the Creative Commons Attribution (CC BY) license (http://creativecommons.org/licenses/by/4.0/). 